\title{
@creative
}

\section{Spherical folding tessellations by kites and isosceles triangles IV}

\author{
Catarina P. Avelino, Altino F. Santos \\ Pólo CMAT-UTAD, Centro de Matemática da Universidade do Minho \\ Universidade de Trás-os-Montes e Alto Douro, UTAD, Quinta de Prados, 5000-801 Vila \\ Real, Portugal
}

Received 13 July 2014, accepted 28 September 2014, published online 18 September 2015

\begin{abstract}
The classification of the dihedral folding tessellations of the sphere and the plane whose prototiles are a kite and an equilateral triangle were obtained in [1]. Recently, this classification was extended to isosceles triangles so that the classification of spherical folding tesselations by kites and isosceles triangles in three cases of adjacency was presented in $[2,3,4]$. In this paper we finalize this classification presenting all the dihedral folding tessellations of the sphere by kites and isosceles triangles in the remaining three cases of adjacency, that consists of five sporadic isolated tilings. A list containing these tilings including its combinatorial structure is presented in Table 1.
\end{abstract}

Keywords: Dihedral f-tilings, combinatorial properties, spherical trigonometry, symmetry groups.

Math. Subj. Class.: 52C20, 05B45, 52B05

\section{Introduction}

By a folding tessellation or folding tiling of the Euclidean sphere $S^{2}$ we mean an edge-toedge pattern of spherical geodesic polygons that fills the whole sphere with no gaps and no overlaps, and such that the "underlying graph" has even valency at any vertex and the sums of alternate angles around each vertex are $\pi$.

Folding tilings ( $f$-tiling, for short) are strongly related to the theory of isometric foldings on Riemannian manifolds. In fact, the set of singularities of any isometric folding corresponds to a folding tiling, see [13] for the foundations of this subject.

The study of this special class of tessellations was initiated in [5] with a complete classification of all spherical monohedral folding tilings. Ten years latter Ueno and Agaoka

E-mail addresses: cavelino@utad.pt (Catarina P. Avelino), afolgado@utad.pt (Altino F. Santos) 
[14] have established the complete classification of all triangular spherical monohedral tilings (without any restriction on angles).

Dawson has also been interested in special classes of spherical tilings, see [10], [11] and [12], for instance.

The complete classification of all spherical folding tilings by rhombi and triangles was obtained in 2005 [8]. A detailed study of the triangular spherical folding tilings by equilateral and isosceles triangles is presented in [9].

Spherical f-filings by two noncongruent classes of isosceles triangles in a particular case of adjacency were recently obtained [6].

Here we discuss dihedral folding tessellations by spherical kites and isosceles spherical triangles.

A spherical kite $K$ (Figure 1(a)) is a spherical quadrangle with two congruent pairs of adjacent sides, but distinct from each other. Let us denote by $\left(\alpha_{1}, \alpha_{2}, \alpha_{1}, \alpha_{3}\right), \alpha_{2}>\alpha_{3}$, the internal angles of $K$ in cyclic order. The length sides are denoted by $a$ and $b$, with $a<b$. From now on $T$ denotes a spherical isosceles triangle with internal angles $\beta$ and $\gamma$ $(\gamma \neq \beta)$, and length sides $c$ and $d$, see Figure 1(b).

We shall denote by $\Omega(K, T)$ the set, up to isomorphism, of all dihedral folding tilings of $S^{2}$ whose prototiles are $K$ and $T$.

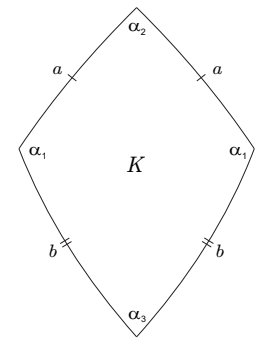

(a)

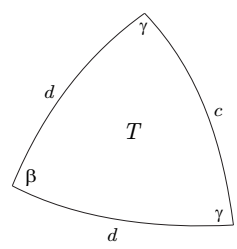

(b)

Figure 1: A spherical kite and a spherical isosceles triangle

Taking into account the area of the prototiles $K$ and $T$ we have

$$
2 \alpha_{1}+\alpha_{2}+\alpha_{3}>2 \pi \text { and } \beta+2 \gamma>\pi .
$$

As $\alpha_{2}>\alpha_{3}$ we also have

$$
\alpha_{1}+\alpha_{2}>\pi
$$

We begin by pointing out that any element of $\Omega(K, T)$ has at least two cells congruent to $K$ and $T$, respectively, such that they are in adjacent positions and in one and only one of the situations illustrated in Figure 2.

After certain initial assumptions are made, it is usually possible to deduce sequentially the nature and orientation of most of the other tiles. Eventually, either a complete tiling or an impossible configuration proving that the hypothetical tiling fails to exist is reached. In the diagrams that follow, the order in which these deductions can be made is indicated by the numbering of the tiles. For $j \geq 2$, the location of tiling $j$ can be deduced directly from the configurations of tiles $(1,2, \ldots, j-1)$ and from the hypothesis that the configuration is part of a complete tiling, except where otherwise indicated. 


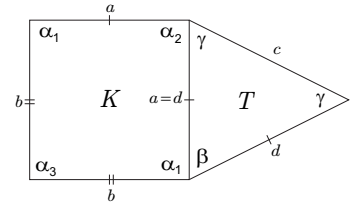

I

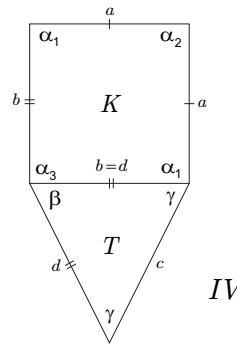

IV

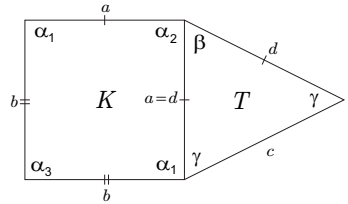

II

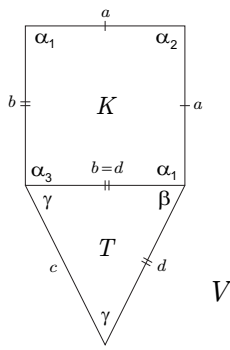

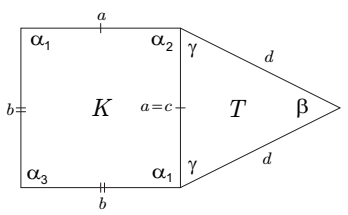

III

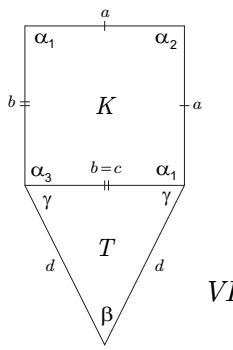

Figure 2: Distinct cases of adjacency of $K$ and $T$

The cases of adjacency $I, I I$ and $I I I$ have already been analyzed in [2, 3, 4]. In this paper we consider the remaining cases of adjacency $I V, V$ and $V I$.

\section{Case of Adjacency IV}

Suppose that any element of $\Omega(K, T)$ has at least two cells congruent, respectively, to $K$ and $T$, such that they are in adjacent positions as illustrated in Figure $2-I V$. As $b=d$, using trigonometric formulas, we obtain

$$
\frac{\cos \gamma(1+\cos \beta)}{\sin \gamma \sin \beta}=\frac{\cos \frac{\alpha_{2}}{2}+\cos \alpha_{1} \cos \frac{\alpha_{3}}{2}}{\sin \alpha_{1} \sin \frac{\alpha_{3}}{2}} .
$$

Concerning the angles of the triangle $T$ we have necessarily one of the following situations:

$$
\gamma>\beta \text { or } \gamma<\beta
$$

In the following subsections we consider separately each one of these cases.

\section{$2.1 \gamma>\beta$}

In this case we have $\gamma>\frac{\pi}{3}$ and $a, c<b=d$.

Proposition 2.1. Under the conditions assumed in this section, there is a single folding tiling, $\mathcal{L}$, such that $\alpha_{2}=\frac{\pi}{2}, \alpha_{1}+\gamma=\pi$ and $\alpha_{3}=\beta=\frac{\pi}{3}$. Planar and $3 D$ representations of $\mathcal{L}$ are given in Figure 9.

Proof. Suppose that any element of $\Omega(K, T)$ has at least two cells congruent, respectively, to $K$ and $T$, such that they are in adjacent positions as illustrated in Figure 2-IV. 
Observing Figure 3(a), tile 3 cannot be a kite - this case was already analyzed in [4] (case 2.1) and, under the current conditions, give rise to no f-tilings. Consequently, a side of length $c$ of each triangle must be adjacent to a side of length $c$ of another triangle. Moreover, we have $\alpha_{1} \geq \alpha_{2}>\alpha_{3}$. In fact, if $\alpha_{2}>\alpha_{1}$ and

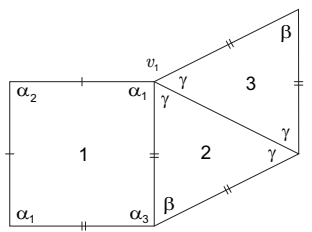

(a)

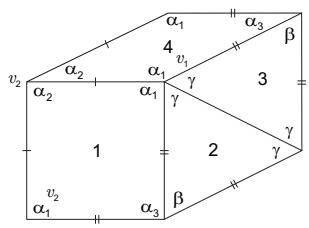

(b)

Figure 3: Local configurations

(i) $\alpha_{1}+\gamma=\pi$ (Figure 3(b)), we reach a contradiction at vertex $v_{2}$, as $\alpha_{2}+\rho>\pi$, for all $\rho \in\left\{\alpha_{1}, \alpha_{2}\right\}$

(ii) $\alpha_{1}+\gamma<\pi$ (Figure 4(a)), at vertex $v_{1}$ we have necessarily $\alpha_{2}+\gamma+k \alpha_{3}=\pi, k \geq 1$. But $\left(\alpha_{1}+\alpha_{1}+\gamma\right)+\left(\alpha_{2}+\gamma+\alpha_{3}\right)>\left(2 \alpha_{1}+\alpha_{2}+\alpha_{3}\right)>2 \pi$, which is impossible.

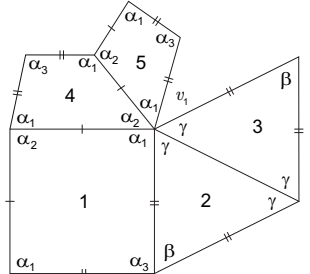

(a)

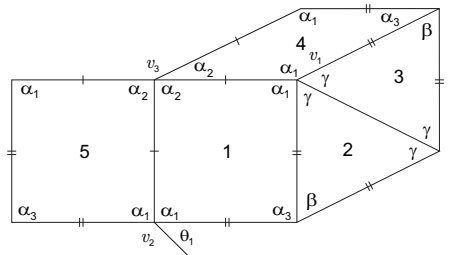

(b)

Figure 4: Local configurations

At vertex $v_{1}$ we have $\alpha_{1}+\gamma=\pi$ or $\alpha_{1}+\gamma<\pi$.

1. Suppose firstly that $\alpha_{1}+\gamma=\pi$ (Figure 4(b)). At vertex $v_{3}$ we have $k \alpha_{2}=\pi$, with $k \geq 2$. As $\left(\alpha_{1}+\gamma\right)+\left(\alpha_{2}+\alpha_{2}+\alpha_{2}\right)>\left(\alpha_{1}+\alpha_{2}\right)+(\gamma+\beta+\beta)>2 \pi$, it follows that $k=2\left(\alpha_{2}=\frac{\pi}{2}\right)$. With the labeling of Figure $4(\mathrm{~b})$, if

(i) $\theta_{1}=\alpha_{3}$ (Figure 5(a)), then at vertex $v_{3}$ we have necessarily $\alpha_{1}+k \beta=\pi, k \geq 2$, and so $\alpha_{1}>\frac{\pi}{2}=\alpha_{2}>\gamma \geq \alpha_{3}>\beta$ (note that $\alpha_{1}+\beta+\alpha_{3}>\pi$ ). But then tile 9 must be a triangle, which is impossible;

(ii) $\theta_{1}=\beta$ (Figure 5(b)), then at vertex $v_{2}$ it follows that $\alpha_{1}+k \beta=\pi, k \geq 2$ (note that we must have $\alpha_{3}>\beta$ ). But at vertex $v_{3}$ we have $\gamma+\gamma<\pi$ and $\gamma+\gamma+\rho>\pi$, for all $\rho$.

(iii) $\theta_{1}=\gamma$, we get the configuration illustrated in Figure 6(a). Now, if 


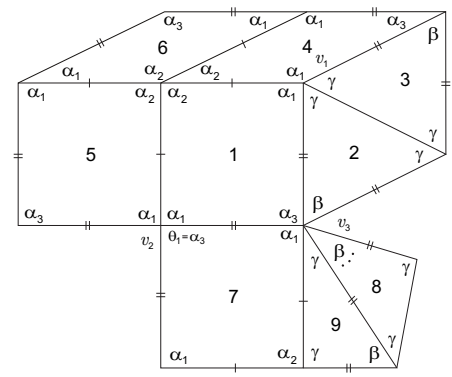

(a) case 1(i)

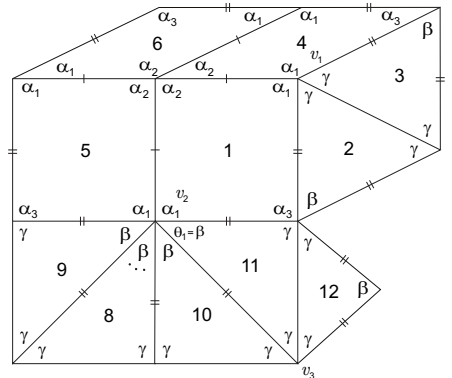

(b) case 1 (ii)

Figure 5: Local configurations

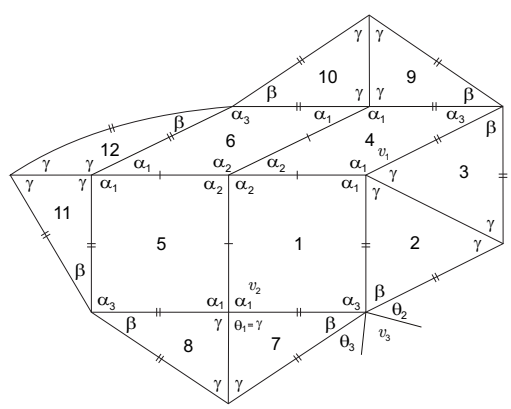

(a) case 1(iii)

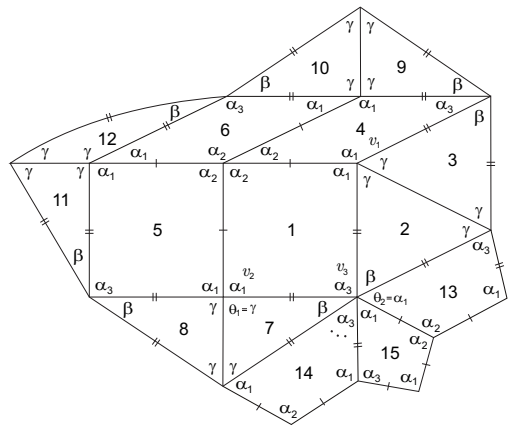

(b) case 1(iii)(a)

Figure 6: Local configurations

(a) $\theta_{2}=\alpha_{1}$ (Figure 6(b)), we have necessarily $\alpha_{1}+k \alpha_{3}=\pi$, with $k \geq 2$, and $\alpha_{1}>\frac{\pi}{2}=\alpha_{2}>\gamma>\beta>\alpha_{3}\left(\alpha_{1}+\beta+\alpha_{3}>\pi\right)$. But then, the other sum of alternate angles at vertex $v_{3}$ must be greater or equal than $\alpha_{1}+\beta+\beta>\pi$, which is a contradiction $\left(3 \pi \geq\left(\alpha_{1}+\gamma\right)+\left(\alpha_{2}+\alpha_{2}\right)+\left(\alpha_{1}+\beta+\beta\right)>\right.$ $\left.\left(2 \alpha_{1}+\alpha_{2}+\alpha_{3}\right)+(\beta+\gamma+\gamma)>3 \pi\right)$.

(b) $\theta_{2}=\beta$ (Figure 7(a)), at vertex $v_{3}$ we have $\gamma+\gamma+k \alpha_{3}=\pi, k \geq 1$, and a contradiction arises at vertex $v_{4}$ as $\alpha_{1}+\rho>\pi$, for all $\rho \in\left\{\alpha_{1}, \alpha_{2}\right\}$.

(c) $\theta_{2}=\gamma$, at vertex $v_{3}$ we have $\theta_{3} \in\left\{\beta, \alpha_{3}\right\}$. In the first case, illustrated in Figure 7(b), we reach a contradiction at vertex $v_{5}$. On the other hand, if $\theta_{3}=$ $\alpha_{3}$, due to the angles involved in the sums of alternate angles at vertex $v_{3}$, we must have $\alpha_{3}=\beta$. Taking into account the triangle and the kite's areas, it follows that $\gamma+\beta+\beta=\gamma+\alpha_{3}+\alpha_{3}=\pi$ (Figure 8). At vertex $v_{6}$ we have $\alpha_{1}+\beta<\pi$ and $\alpha_{1}+\beta+\rho>\pi$, for all $\rho \in\left\{\alpha_{1}, \alpha_{2}, \alpha_{3}, \beta, \gamma\right\}$.

(d) $\theta_{2}=\alpha_{3}$, taking into account the analysis of the previous cases, at vertex $v_{3}$ we have $k \alpha_{3}=k \beta=\pi, k \geq 3$. Due to the kite's area, it follows that $\gamma-$ $\frac{\pi}{4}<\frac{\beta}{2}$ and consequently $\cos \frac{\beta}{2}<\cos \left(\gamma-\frac{\pi}{4}\right)$. Using equation (2.1), we conclude that $\beta>\frac{\pi}{4}$, and so $k=3$. The last configuration is then extended to the one illustrated in Figure 9(a). We shall denote this f-tiling by $\mathcal{L}$. Its $3 \mathrm{D}$ 


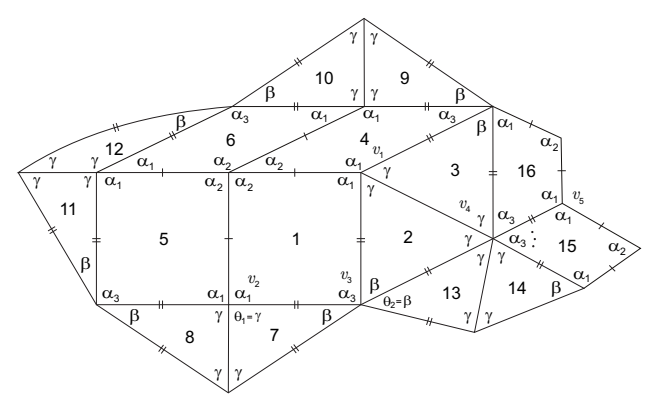

(a) case 1(iii)(b)

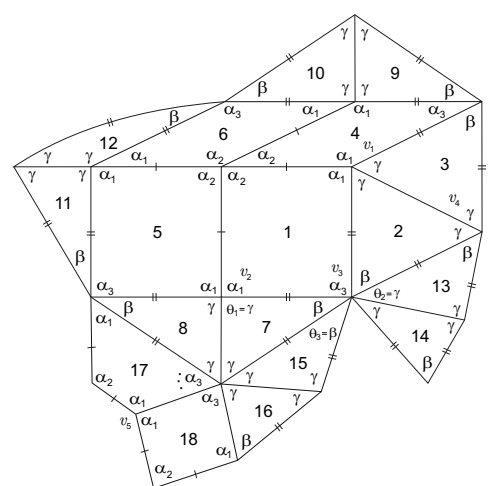

(b) case 1(iii)(c)

Figure 7: Local configurations

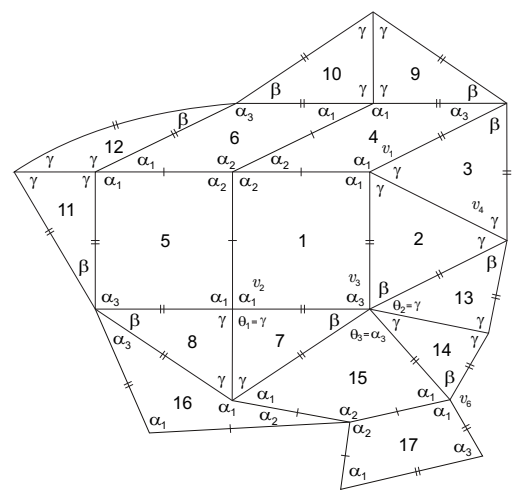

Figure 8: Local configuration occurring in case 1(iii)(c)

representation is given in Figure 9(b).

2. Suppose now that $\alpha_{1}+\gamma<\pi$ (Figure 3(a)). Again, due to the analysis made in [4] (case 2.1), we use the fact that a side of length $c$ of each triangle must be adjacent to a side of length $c$ of other triangle. At vertex $v_{1}$ we must have $\alpha_{1}+\gamma+k \alpha_{3}=\pi$, with $k \geq 1$. Nevertheless, we reach a contradiction at vertex $v_{2}$ (Figure 10) since there is no way to satisfy the angle-folding relation around this vertex.

\section{$2.2 \gamma<\boldsymbol{\beta}$}

In this case we have $\beta>\frac{\pi}{3}$ and $a<b=d<c$.

Proposition 2.2. Under the conditions assumed in this section, there is a single folding tiling, $\mathcal{J}$, such that $\alpha_{2}=\frac{\pi}{2}, \alpha_{1}+\gamma=\pi, \gamma=\frac{\pi}{3}$ and $\beta+\beta+\alpha_{3}=\pi$. Planar and $3 D$ representations of $\mathcal{J}$ are given in Figure 12. 


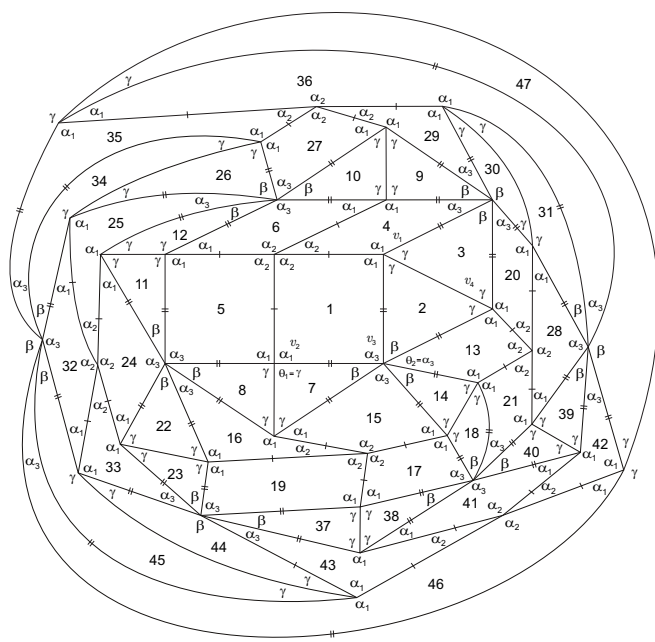

(a) planar representation

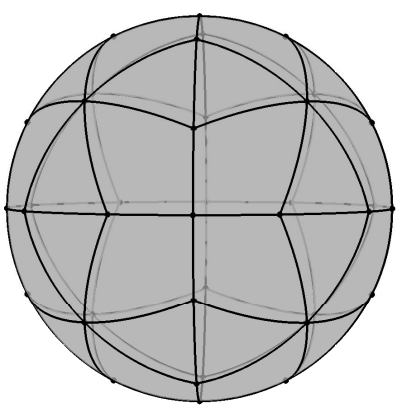

(b) $3 \mathrm{D}$ representation

Figure 9: f-tiling $\mathcal{L}$

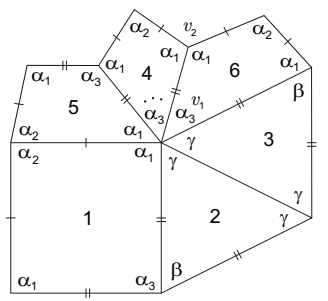

Figure 10: Local configuration occurring in case 2

Proof. Suppose that any element of $\Omega(K, T)$ has at least two cells congruent, respectively, to $K$ and $T$, such that they are in adjacent positions as illustrated in Figure $2-I V$. As $a \neq c$, we get the configuration illustrated in Figure 11(a), and, at vertex $v_{1}$, we have

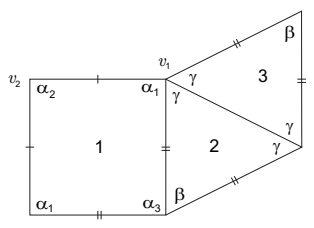

(a)

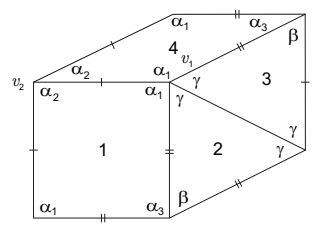

(b) case 1

Figure 11: Local configurations 
$\alpha_{1}+\gamma=\pi$ or $\alpha_{1}+\gamma<\pi$.

1. Suppose firstly that $\alpha_{1}+\gamma=\pi$ (Figure 11(b)).

Note that the conditions $\alpha_{2}>\alpha_{1} \geq \alpha_{3}$ and $\alpha_{2}>\alpha_{3}>\alpha_{1}$ lead to a contradiction at vertex $v_{2}$, as $\alpha_{2}+\rho>\pi$, for all $\rho \in\left\{\alpha_{1}, \alpha_{2}\right\}$. Therefore $\alpha_{1} \geq \alpha_{2}>\alpha_{3}$. Now, if

(i) $\alpha_{2}+\alpha_{2}=\pi$, then $\beta+\beta+k \alpha_{3}=\pi, k \geq 1$, and so $\alpha_{1}>\alpha_{2}=\frac{\pi}{2}>\beta>\gamma>\alpha_{3}$. Consequently, $\gamma=\frac{\pi}{3}$ (as $\beta<\frac{\pi}{2}$, we have $\gamma>\frac{\pi}{4}$ ). Then, the last configuration is extended to the one illustrated in Figure 12(a). We shall denote this f-tiling by $\mathcal{J}$. Its $3 \mathrm{D}$ representation is given in Figure 12(b).

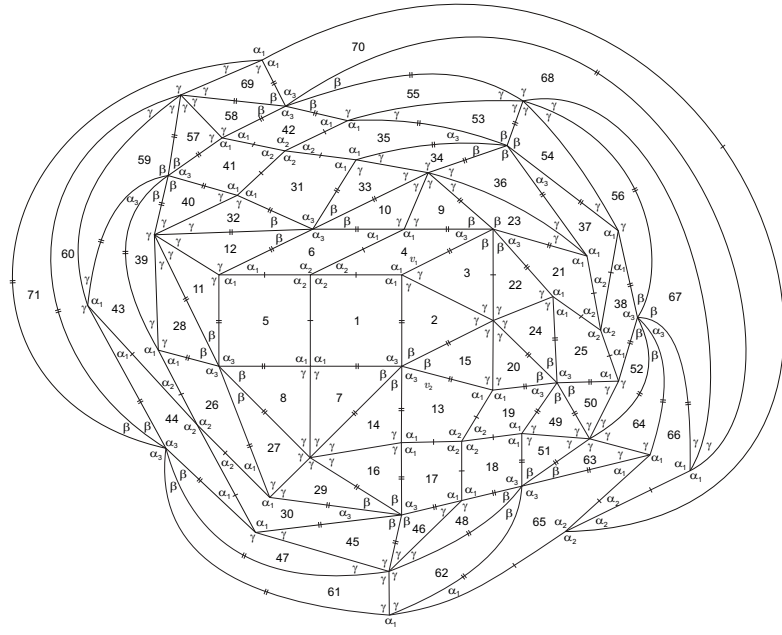

(a) planar representation

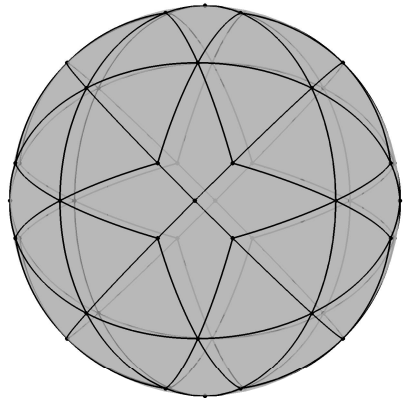

(b) $3 \mathrm{D}$ representation

Figure 12: f-tiling $\mathcal{J}$

(ii) $\alpha_{2}+\alpha_{2}<\pi$, then $k \alpha_{2}=\pi, k \geq 3, \beta+\beta+\bar{k} \alpha_{3}=\pi, \bar{k} \geq 1$, and so $\alpha_{1}>\frac{\pi}{2}>$ $\beta>\alpha_{2}>\gamma>\alpha_{3}$. As $\gamma>\frac{\pi}{4}$, we have necessarily $k=3$ (Figure 13(a)). Now, if at vertex $v_{2}$ we have $\bar{k}>1$ (Figure 13(b)), one of the angles $\theta_{2}, \theta_{3}$ or $\theta_{4}$ must be $\alpha_{3}$. But then we reach a contradiction at vertex $v_{3}, v_{4}$ or $v_{5}$, respectively, as $\alpha_{1}+\rho>\pi$, for all $\rho \in\left\{\alpha_{1}, \alpha_{2}\right\}$. On the other hand, if $\bar{k}=1$, we get the configuration illustrated in Figure 14(a) (note that at vertex $v_{3}$ we cannot have $\gamma+\gamma+\gamma=\pi$, as $\frac{\pi}{3}=\alpha_{2}>\gamma$ ). At vertex $v_{4}$ we reach a similar contradiction as in the case $\bar{k}>1$.

2. Suppose now that $\alpha_{1}+\gamma<\pi$ (Figure 11(a)).

If $\alpha_{2}>\alpha_{1} \geq \alpha_{3}$ or $\alpha_{2}>\alpha_{3}>\alpha_{1}$, at vertex $v_{1}$ we must have $\alpha_{2}+k \gamma=\pi$, with $k \geq 2$, and consequently at vertex $v_{2}$ it follows that $\alpha_{1}+\alpha_{1} \leq \pi$, and so $\alpha_{1} \leq \frac{\pi}{2}$ and $\alpha_{2}+\alpha_{3}>\pi$. But then an incompatibility on the sides arises at vertex $v_{1}$.

If $\alpha_{1} \geq \alpha_{2}>\alpha_{3}$, and

(i) $\theta_{1}=\alpha_{3}$ (Figure 14(b)), then $\theta_{2}$ must be $\beta$, otherwise we get, at vertex $v_{3}, \theta_{3}=\alpha_{1}$ and $\alpha_{1}+\rho>\pi$, for all $\rho \in\left\{\alpha_{1}, \alpha_{2}\right\}$. Nevertheless, an impossibility cannot be avoided at vertex $v_{1}$ since we obtain $\beta+\gamma+\rho>\pi$, for all $\rho \in\left\{\alpha_{1}, \alpha_{2}\right\}$. 


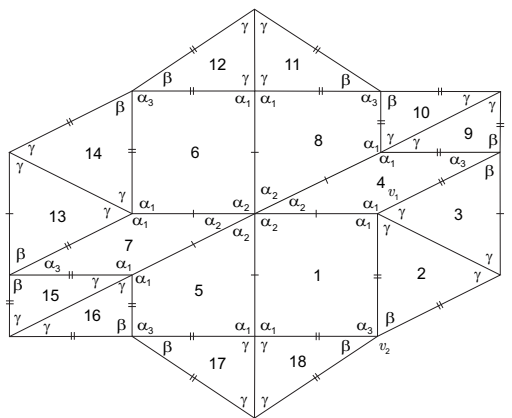

(a)

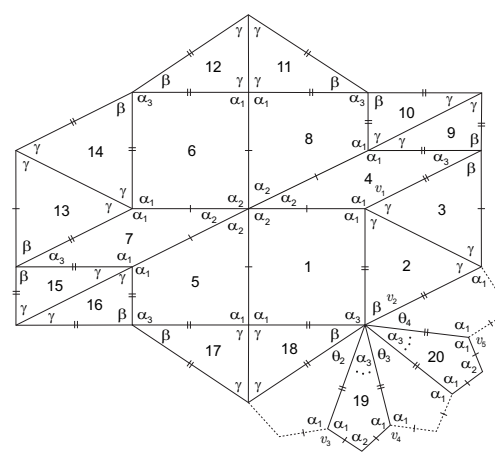

(b)

Figure 13: Local configurations occurring in case 1(ii)

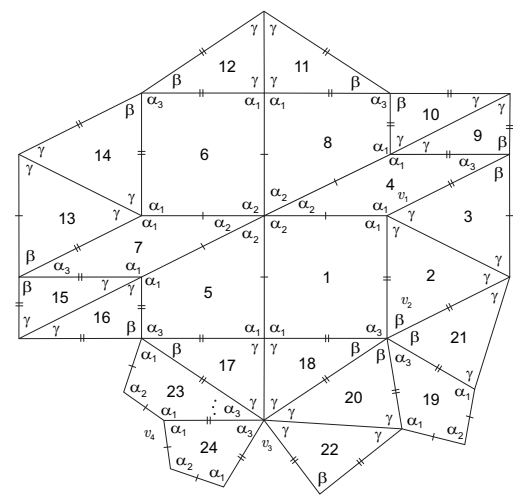

(a) case 1(ii)

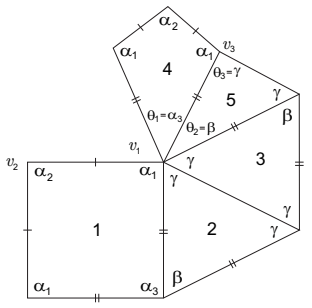

(b) case 2(i)

Figure 14: Local configurations

(ii) $\theta_{1}=\gamma$ and

(a) $\theta_{2}=\beta$ (Figure 15(a)), then $\gamma<\frac{\pi}{4}$ and $\beta>\frac{\pi}{2}$. At vertex $v_{4}$ we must have $\beta+\alpha_{2} \leq \pi$, however $2 \pi \geq\left(\alpha_{1}+\gamma+\gamma\right)+\left(\beta+\alpha_{2}\right)=(\beta+\gamma+\gamma)+\left(\alpha_{1}+\alpha_{2}\right)>$ $2 \pi$ is impossible.

(b) $\theta_{2}=\gamma$, it follows that $\alpha_{1}+k \gamma=\pi, k \geq 2$, as illustrated in Figure 15(b). But any choices for $\theta_{3}$ and $\theta_{4}$ lead to a contradiction.

\section{Case of Adjacency $V$}

Proposition 3.1. $\Omega(K, T)$ is composed by a single folding tiling, $\mathcal{M}$, such that $\alpha_{2}=\frac{\pi}{2}$, $\alpha_{1}+\beta=\pi$ and $\gamma=\alpha_{3}=\frac{\pi}{3}$. For a planar representation see Figure 20(b). Its $3 D$ representation is given in Figure 21 . 


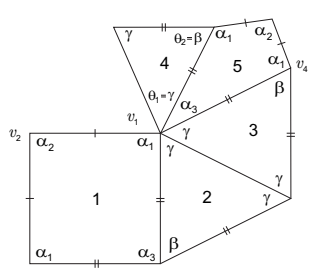

(a)

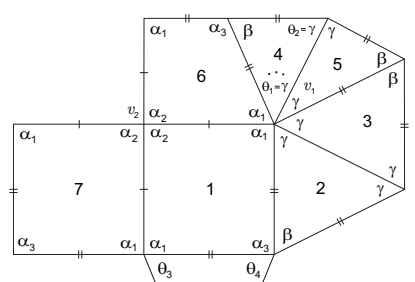

(b)

Figure 15: Local configurations occurring in case 2(ii)

Proof. Suppose that any element of $\Omega(K, T)$ has at least two cells congruent, respectively, to $K$ and $T$, such that they are in adjacent positions as illustrated in Figure 2- $V$.

The case analyzed in [4] (case 2.1) give rise to no f-tilings including two cells in these adjacent positions, and so a side of length $c$ of each triangle must be adjacent to a side of length $c$ of another triangle.

1. If $\alpha_{2}>\alpha_{1}$, then $\alpha_{2}>\frac{\pi}{2}$ and we get the configuration of Figure 16(a).

If $\alpha_{2}+\beta=\pi$ (Figure 16(b)), we have $\alpha_{1}=\frac{\pi}{2}$ (vertex $v_{1}$ ), and so $\alpha_{2}+\alpha_{3}>\pi$, justifying the choice for $\theta_{1}$. But at vertex $v_{2}$ we obtain a contradiction as $\alpha_{3}+\gamma+\gamma>\pi$ $\left(\left(\alpha_{1}+\alpha_{1}\right)+\left(\alpha_{2}+\beta\right)+\left(\alpha_{3}+\gamma+\gamma\right)=\left(2 \alpha_{1}+\alpha_{2}+\alpha_{3}\right)+(\beta+\gamma+\gamma)>3 \pi\right)$.

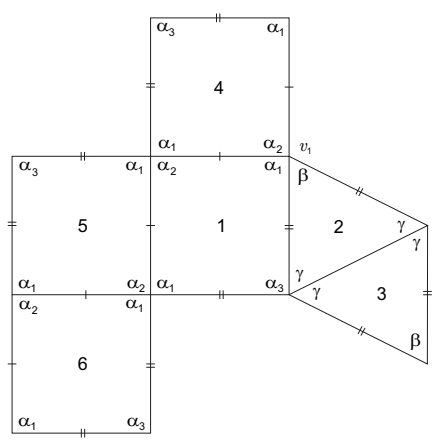

(a)

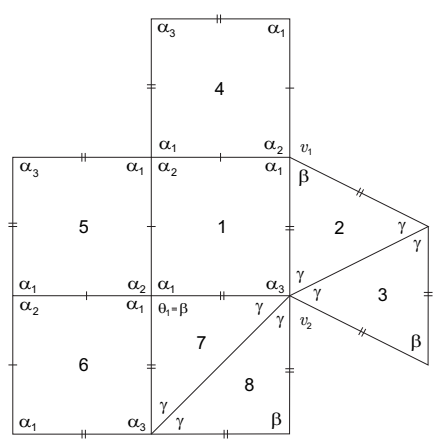

(b)

Figure 16: Local configurations occurring in case 1

If $\alpha_{2}+\beta<\pi$, then $\alpha_{2}+k \beta=\pi$, with $k \geq 2$ (note that $\alpha_{2}+\alpha_{3}>\pi$ ). Consequently, $\gamma>\beta$ and $\alpha_{3}>\beta$. Observing Figure 17(a), we conclude that there is no way to satisfy the angle-folding relation around vertex $v_{2}\left(\alpha_{2}+\alpha_{2}>\alpha_{2}+\alpha_{1}>\pi, \alpha_{2}+\alpha_{3}>\pi\right.$, $\alpha_{2}+\gamma+\rho>\pi$, for all $\rho$, and $\theta_{1}=\beta$ implies $\theta_{2}=\gamma$ and $\gamma+\gamma+\rho>\pi$, for all $\rho$ ).

2. Suppose now that $\alpha_{1} \geq \alpha_{2}$ (Figure 17(b)). It follows that $\alpha_{1}>\frac{\pi}{2} \geq \alpha_{2}>\beta$ and $\gamma>\frac{\pi}{4}$.

2.1 If $\beta>\gamma$, then at vertex $v_{1}$ we must have $\alpha_{1}+\beta+k \alpha_{3}=\pi$, with $k \geq 1$, or $\alpha_{1}+\beta=\pi$.

In the first case we have $\alpha_{1}>\frac{\pi}{2} \geq \alpha_{2}>\beta>\gamma>\alpha_{3}$ (Figure 18(a)). As $\theta_{1}$ or $\theta_{2}$ must be $\alpha_{3}$, we get an impossibility at vertex $v_{2}$ or $v_{3}$, respectively. 


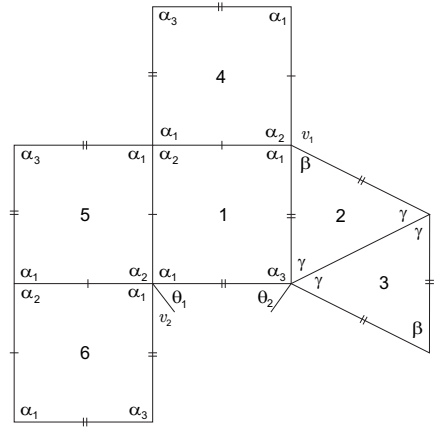

(a) case 1

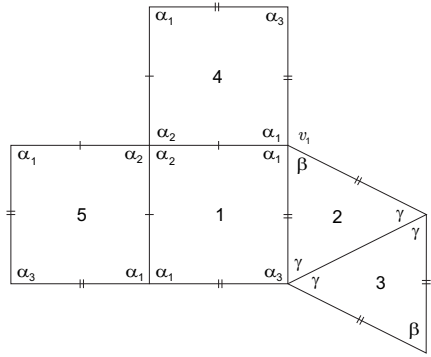

(b) case 2

Figure 17: Local configurations

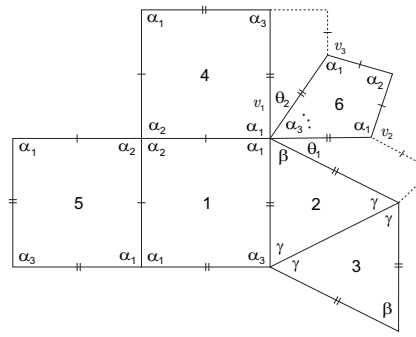

(a)

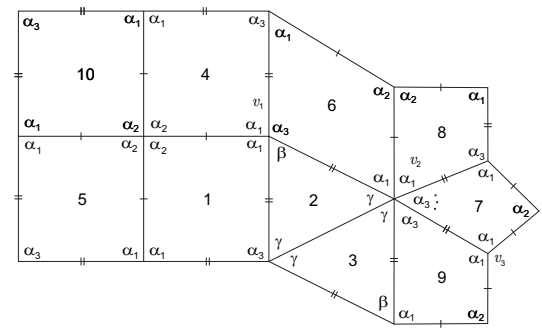

(b)

Figure 18: Local configurations occurring in case 2.1

Therefore $\alpha_{1}+\beta=\pi$. At vertex $v_{1}$ we cannot have $\alpha_{1}+\beta=\pi=\alpha_{1}+\alpha_{3}$, as illustrated in Figure 18(b), otherwise at vertex $v_{2}$ we get $\alpha_{1}+\gamma+k \alpha_{3}=\pi, k \geq 1$, and a contradiction arises at vertex $v_{3}$. Consequently, we get the configuration illustrated in Figure 19(a). Note

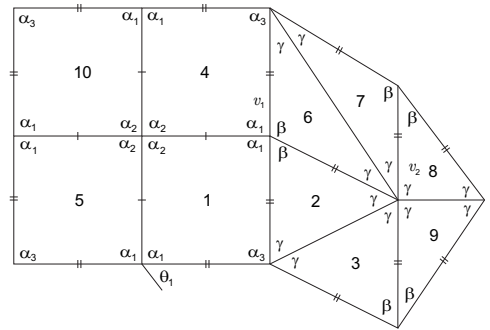

(a)

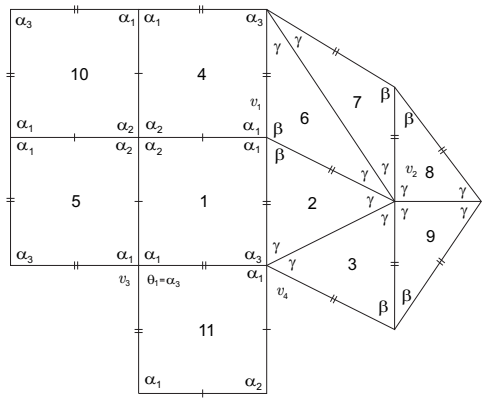

(b)

Figure 19: Local configurations occurring in case 2.1

that at vertex $v_{2}$ we cannot have $\gamma+\gamma+k \alpha_{3}=\pi, k \geq 1$, nor $\gamma+\gamma+\gamma+k \alpha_{3}=\pi, k \geq 1$, 
otherwise we obtain a similar contradiction as before (in fact we cannot have two angles $\alpha_{3}$ adjacent). Observe also that we have necessarily $\alpha_{2}+\alpha_{2}=\pi$, as $\alpha_{2}+\alpha_{2}+\alpha_{2}>\pi$.

Now, $\theta_{1}=\alpha_{3}, \theta_{1}=\gamma$ or $\theta_{1}=\beta$.

2.1.1 If $\theta_{1}=\alpha_{3}$ (Figure 19(b)), at vertex $v_{3}$ we must have $\alpha_{1}+\alpha_{3}=\pi$, which implies $\alpha_{3}=\beta$. Nevertheless, a contradiction arises at vertex $v_{4}$ since we get $\alpha_{1}+\gamma+k \alpha_{3}>\pi$, for all $k \geq 1$.

2.1.2 If $\theta_{1}=\gamma$ (Figure 20(a)), at vertex $v_{4}$ we obtain $\beta+\gamma+k \alpha_{3}=\pi$. But at vertex $v_{3}$ we get $\alpha_{1}+\gamma+\bar{k} \alpha_{3}=\pi$, which is not possible as $3 \pi \geq\left(\alpha_{1}+\gamma+\alpha_{3}\right)+\left(\alpha_{1}+\beta\right)+\left(\alpha_{2}+\alpha_{2}\right)>$ $\left(2 \alpha_{1}+\alpha_{2}+\alpha_{3}\right)+(\beta+\gamma+\gamma)>3 \pi$.

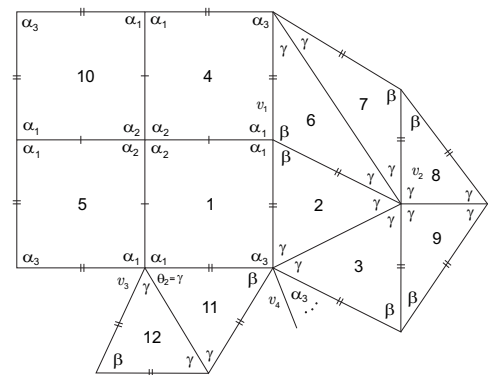

(a)

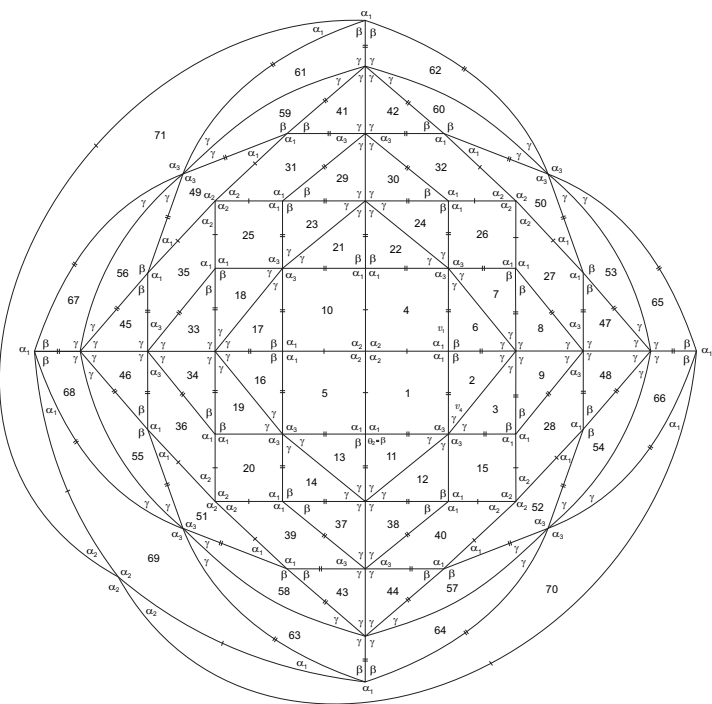

(b) f-tiling $\mathcal{M}$

Figure 20: Local configurations occurring in case 2.1

2.1.3 If $\theta_{1}=\beta$, the last configuration is extended to the one illustrated in Figure 20(b). We shall denote this f-tiling by $\mathcal{M}$. Its 3D representation is given in Figure 21 .

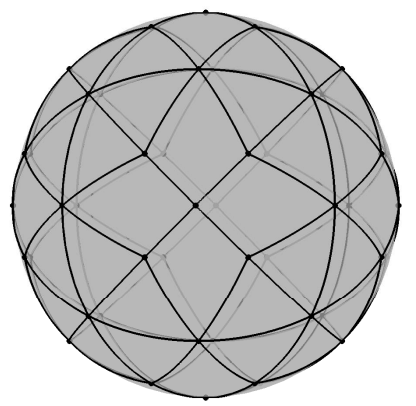

Figure 21: f-tiling $\mathcal{M}$ 
2.2 Suppose now that $\beta<\gamma$ (Figure 22(a)). In this case we have $\gamma>\frac{\pi}{3}$ and $\theta_{1}=\beta$ or $\theta_{1}=\alpha_{3}$.

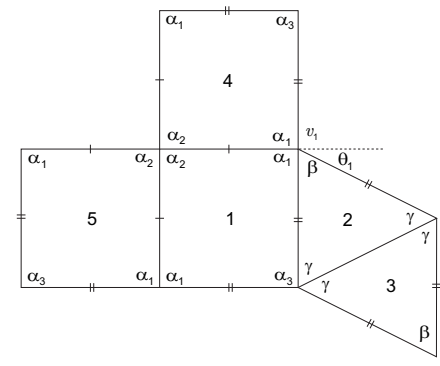

(a)

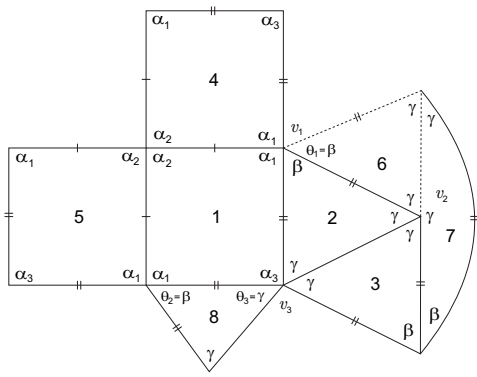

(b)

Figure 22: Local configurations occurring in case 2.2

If $\theta_{1}=\beta\left(\alpha_{1}+\beta \leq \pi\right.$, see Figure 22(b)), then at vertex we must have $\gamma+\gamma+$ $k \alpha_{3}=\pi$, with $k \geq 0$. As we seen before, as two angles $\alpha_{3}$ in adjacent positions lead to a contradiction, we must have $\gamma+\gamma=\pi$. Moreover, $\theta_{2}$ cannot be $\alpha_{3}$, otherwise we would obtain $\theta_{3}=\alpha_{1}$ and, at vertex $v_{3}, \alpha_{1}+\gamma>\pi$. The case $\theta_{2}=\beta$ also leads to a contradiction as $\gamma+\gamma=\pi$ and vertex $v_{3}$ cannot have valency four.

Finally, if $\theta_{1}=\alpha_{3}$, we obtain the configuration illustrated in Figure 23. At vertex $v_{1}$ we reach a contradiction as $\left(\alpha_{1}+\beta+\alpha_{3}\right)+\left(\alpha_{1}+\gamma\right)+\left(\alpha_{2}+\alpha_{2}\right)>\left(2 \alpha_{1}+\alpha_{2}+\alpha_{3}\right)+$ $(\beta+\gamma+\gamma)>3 \pi$.

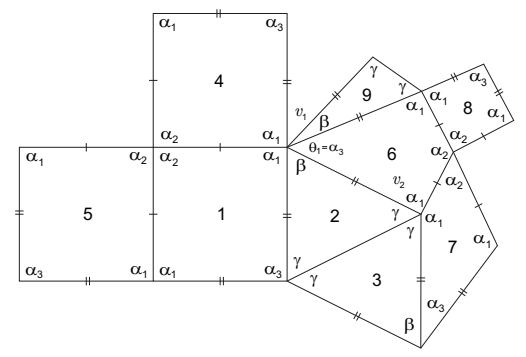

Figure 23: Local configuration occurring in case 2.2

\section{Case of Adjacency VI}

Suppose that any element of $\Omega(K, T)$ has at least two cells congruent, respectively, to $K$ and $T$, such that they are in adjacent positions as illustrated in Figure $2-V I$. As $b=c$, using trigonometric formulas, we obtain

$$
\frac{\cos \beta+\cos ^{2} \gamma}{\sin ^{2} \gamma}=\frac{\cos \frac{\alpha_{2}}{2}+\cos \alpha_{1} \cos \frac{\alpha_{3}}{2}}{\sin \alpha_{1} \sin \frac{\alpha_{3}}{2}} .
$$


Remark 4.1. The cases analyzed in [2] and [3] give rise to no f-tilings including two cells in these adjacent positions, and so a side of length $c$ of each triangle must be adjacent to a side of length $c$ of another triangle.

Proposition 4.2. $\Omega(K, T) \neq \emptyset$ iff

(i) $\alpha_{1}+\gamma=\pi, \alpha_{2}=\frac{\pi}{2}, \gamma+\gamma+\alpha_{3}=\pi$ and $\beta=\frac{\pi}{3}$, or

(ii) $\alpha_{1}+\gamma=\pi, \alpha_{2}=\beta=\frac{\pi}{2}$ and $\gamma+\gamma+\alpha_{3}=\pi$.

In the first case, there is a single f-tiling denoted by $\mathcal{N}$. A planar representation is given in Figure 26(b) and a $3 D$ representation is given in Figure 27.

In the second case, there is a single $f$-tiling, $\mathcal{P}$. The corresponding planar and $3 D$ representations are given in Figure 29(b) and Figure 30, respectively.

Proof. Concerning the angles of the triangle $T$ we have necessarily one of the following situations:

$$
\gamma>\beta \text { or } \gamma<\beta .
$$

We consider separately each one of these cases.

1. Suppose firstly that $\gamma>\beta$.

If $\alpha_{2}>\alpha_{1}$, then $\alpha_{2}>\frac{\pi}{2}$ and we get the configuration of Figure 24(a). Due to the edge

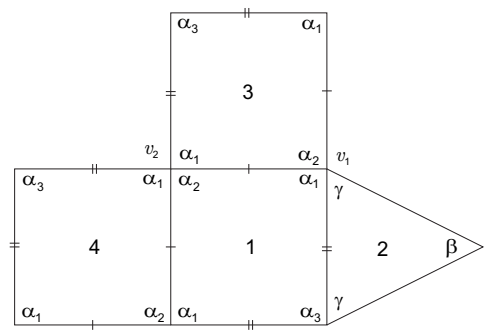

(a)

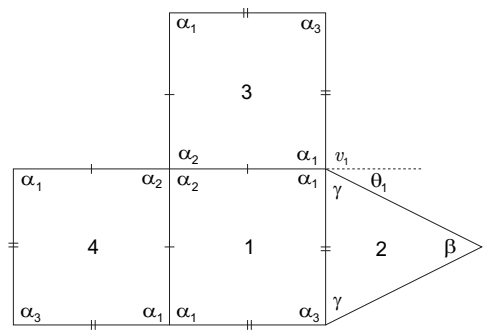

(b)

Figure 24: Local configurations occurring in case 1

lengths and also Remark 4.1, $v_{1}$ cannot have valency four and so $\alpha_{2}+\gamma+k \alpha_{3}=\pi, k \geq 1$. Therefore, analyzing vertices $v_{1}$ and $v_{2}$ we conclude that $\alpha_{2}+\alpha_{3}<\pi$ and $\alpha_{1} \leq \frac{\pi}{2}$, which is impossible taking into account the kite's area.

Thus, $\alpha_{1} \geq \alpha_{2}>\alpha_{3}$ (Figure 24(b)) and $\theta_{1}=\beta$ or $\theta_{1}=\gamma$. In the first case, $v_{1}$ cannot have valency four and there is no way to satisfy the angle-folding relation around this vertex. Consequently, $\theta_{1}=\gamma$ and

(i) if $\alpha_{1}+\gamma<\pi$, then $\alpha_{1}+\gamma+k \alpha_{3}=\pi, k \geq 1$ (Figure 25(a)). At vertex $v_{2}$ we reach a contradiction as $\alpha_{1}+\rho>\pi$, for all $\rho \in\left\{\alpha_{1}, \alpha_{2}\right\}$.

(ii) if $\alpha_{1}+\gamma=\pi$, then the last configuration extends to the one illustrated in Figure 25(b). Now, if $\theta_{2}=\beta$ (Figure 26(a)), we obtain a contradiction at vertex $v_{2}$. On the other hand, if $\theta_{2}=\gamma$ a global planar representation is achieved as illustrated in Figure 26(b). We denote such f-tiling by $\mathcal{N}$. The corresponding 3D representation is given in Figure 27. 


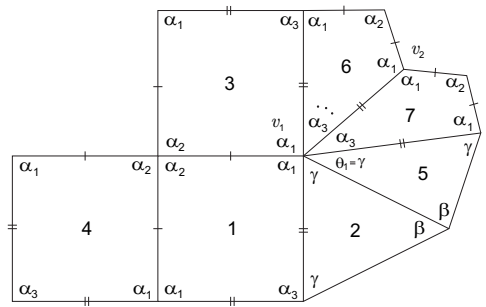

(a) case 1(i)

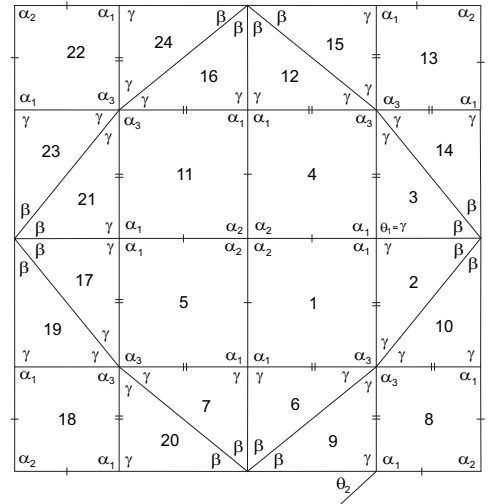

(b) case 1(ii)

Figure 25: Local configurations occurring in case 1

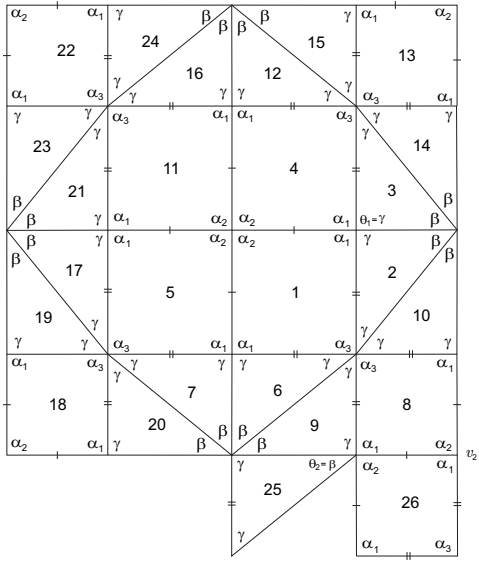

(a)

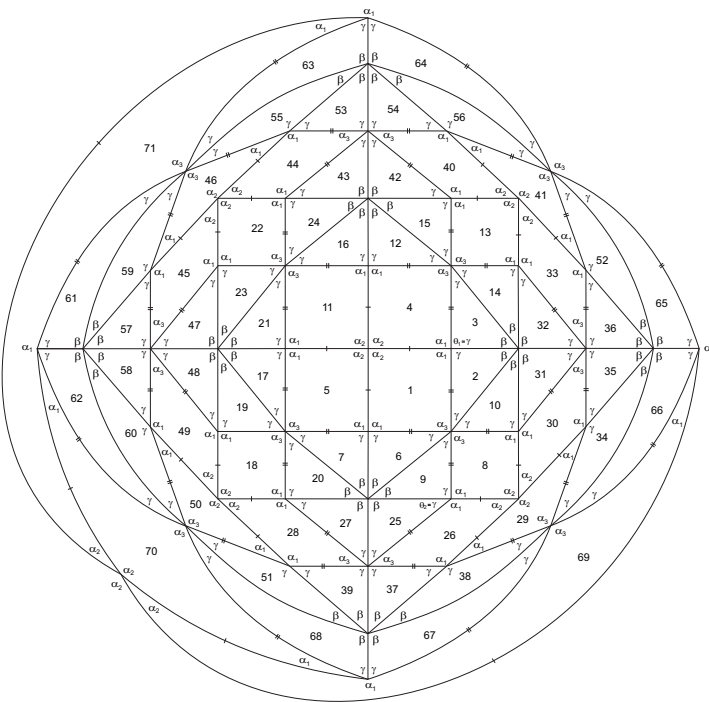

(b) f-tiling $\mathcal{N}$

Figure 26: Local configurations occurring in case 1(ii)

2. Suppose now that $\gamma<\beta$.

If $\alpha_{2}>\alpha_{1}$, then $\alpha_{2}>\frac{\pi}{2}$ and we get the configuration of Figure 28(a). Due to the edge lengths and also Remark 4.1, $v_{1}$ cannot have valency four and so $\alpha_{1}+\alpha_{1}+k \gamma=\pi$, $k \geq 1$. But then the other sum of alternate angles must contain $\alpha_{2}+\alpha_{3}>\pi$, which is not possible. 


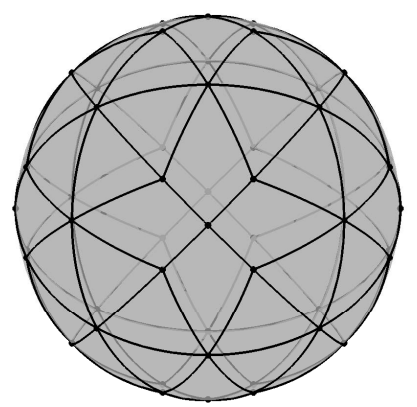

Figure 27: f-tiling $\mathcal{N}$

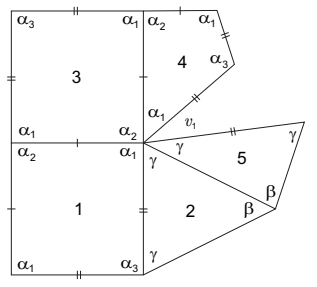

(a)

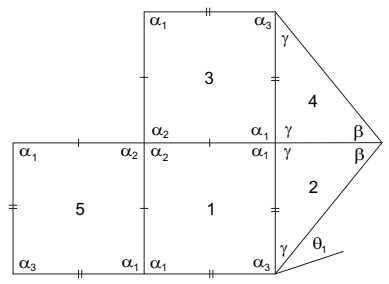

(b)

Figure 28: Local configurations occurring in case 2

Therefore, $\alpha_{1} \geq \alpha_{2}>\alpha_{3}$ and $k \alpha_{2}=\pi, k \geq 2$, and we have $\alpha_{1}+\gamma=\pi$ or $\alpha_{1}+\gamma<\pi$.

2.1 If $\alpha_{1}+\gamma=\pi$, with the labeling of Figure 28(b), we have $\theta_{1}=\gamma$ or $\theta_{1}=\beta$.

2.1.1 If $\theta_{1}=\gamma$, the last configuration is extended to the one illustrated in Figure 29(a).

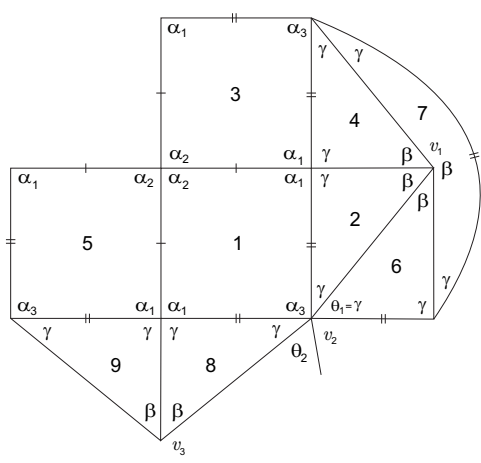

(a)

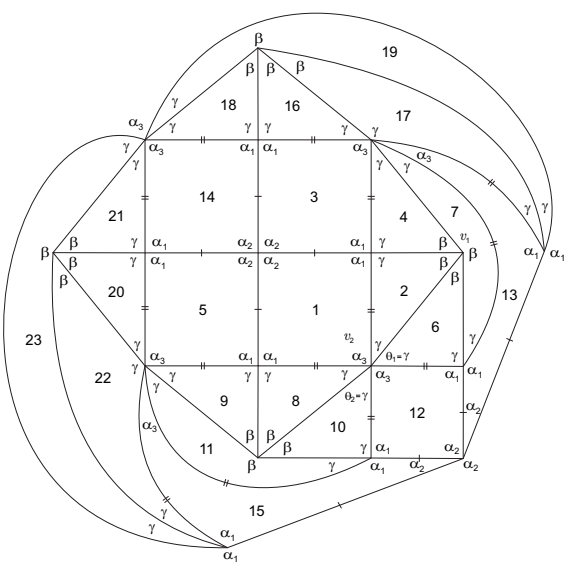

(b) f-tiling $\mathcal{P}$

Figure 29: Local configurations occurring in case 2.1 
2.1.1.1 If $\theta_{2}=\gamma$, at vertex $v_{2}$ we have $\alpha_{3}+\gamma+\gamma=\pi$ or $\alpha_{3}+\gamma+\gamma+\gamma=\pi$. Note that we cannot have more angles $\alpha_{3}$ around $v_{2}$, as two angles of this type in adjacent positions lead to an impossibility, as seen before.

The condition $\alpha_{3}+\gamma+\gamma=\pi$ implies $\alpha_{2}+\alpha_{2}=\frac{\pi}{2}$, and we get the configuration illustrated in Figure 29(b). We denote this f-tiling by $\mathcal{P}$, whose 3D representation is given in Figure 30.

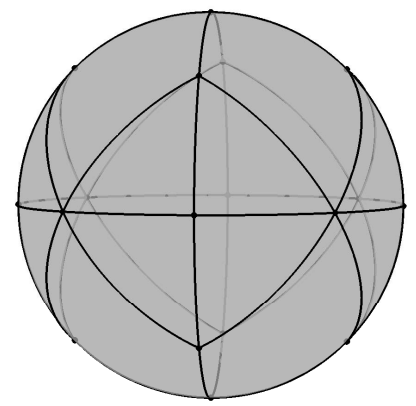

Figure 30: f-tiling $\mathcal{P}$

On the other hand, if $\alpha_{3}+\gamma+\gamma+\gamma=\pi$ (Figure 31(a)), the angles $\theta_{3}$ and $\theta_{4}$ cannot be $\alpha_{3}$ otherwise we reach a contradiction at vertices $v_{3}$ and $v_{4}$, respectively. But this implies that at vertex $v_{5}$ we have two angles $\alpha_{3}$ in adjacent positions, which is not also possible.

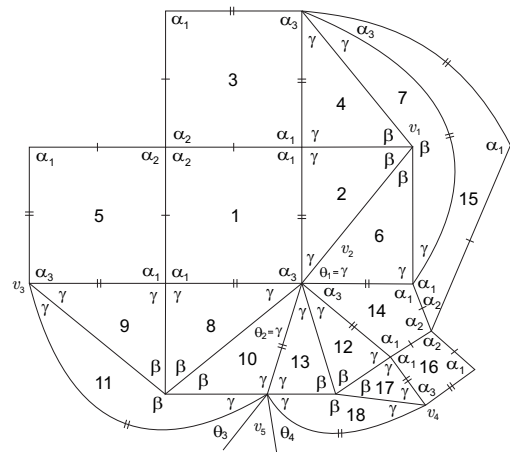

(a)

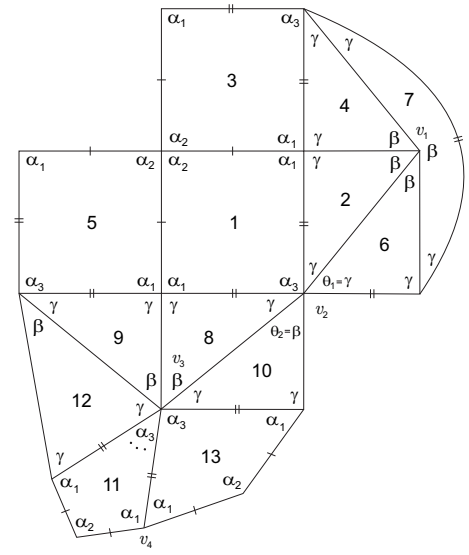

(b)

Figure 31: Local configurations occurring in case 2.1

2.1.1.2 If $\theta_{2}=\beta$, then at vertex $v_{3}$ we have $\beta+\gamma+k \alpha_{3}=\pi, k \geq 1$, which leads to a contradiction as illustrated in Figure 31(b) (see vertex $v_{4}$ ).

2.1.2 If $\theta_{1}=\beta$, we obtain a similar impossibility as in the previous case.

2.2 If $\alpha_{1}+\gamma<\pi$ (Figure 32(a)), then $\theta_{1} \in\{\beta, \gamma\}$. 


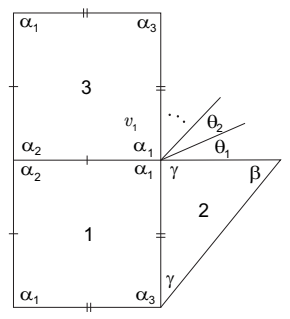

(a)

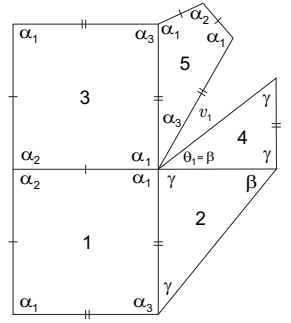

(b)

Figure 32: Local configurations occurring in case 2.2

If $\theta_{1}=\beta$ (Figure 32(b)), then $\alpha_{1}+\beta+k \alpha_{3}=\pi, k \geq 1$. It follows that the other sum of alternate angles at vertex $v_{1}$ must be greater or equal to $\alpha_{1}+\gamma+\gamma>\pi$, which is an impossibility.

If $\theta_{1}=\gamma$ and

(i) $\theta_{2}=\gamma$ (Figure 33(a)), then $\beta>\alpha_{1}>\frac{\pi}{2}$, which implies tile 6. At vertex $v_{2}$ we obtain $\beta+\gamma+k \alpha_{3}=\pi, k \geq 1$, giving rise to two angles $\alpha_{3}$ in adjacent positions, which leads to a contradiction, as seen previously.

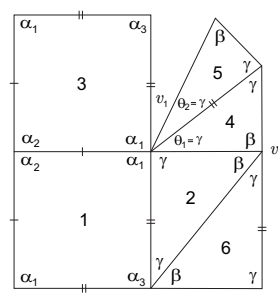

(a)

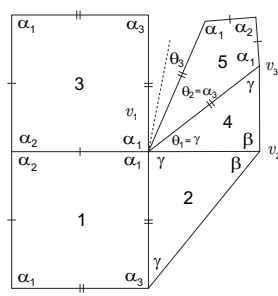

(b)

Figure 33: Local configurations occurring in case 2.2

(ii) $\theta_{2}=\alpha_{3}$ (Figure 33(b)), vertex $v_{1}$ has valency six or greater than six. In the first case, we obtain two angles $\alpha_{3}$ in adjacent positions, which is not possible. In the last case, we have necessarily $\theta_{3}=\gamma$, and so $\beta>\alpha_{1}>\frac{\pi}{2}$. Consequently, a contradiction arises at vertex $v_{2}$ or $v_{3}$. 
Concerning to the combinatorial structure of each tiling obtained, we have that

(i) the symmetries of the f-tilings $\mathcal{L}, \mathcal{J}, \mathcal{M}$ and $\mathcal{N}$ that fix a vertex $v$ of valency four and surrounded by $\left(\alpha_{2}, \alpha_{2}, \alpha_{2}, \alpha_{2}\right)$ are generated by a reflection and by the rotation through an angle $\frac{\pi}{2}$ around the axis by $\pm v$. On the other hand, for any vertices $v_{1}$ and $v_{2}$ of this type, there is a symmetry sending $v_{1}$ into $v_{2}$. It follows that the symmetry group has exactly $48=6 \times 8$ elements and it forms the group of all symmetries of the cube - the octahedral group, sometimes referred as $C_{2} \times S_{4}$.

(ii) the f-tiling $\mathcal{P}$ has only two vertices surrounded by $\left(\alpha_{2}, \alpha_{2}, \alpha_{2}, \alpha_{2}\right)$, say the north and south poles. The symmetries of $\mathcal{P}$ that fix the north pole are generated by a reflection and by the rotation through an angle $\frac{\pi}{2}$ around the $z z$ axis, giving rise to a subgroup isomorphic to $D_{4}$ (the dihedral group of order 8). Now, the reflection on the equator is also a symmetry of $\mathcal{P}$, and so it follows that the symmetry group of $\mathcal{P}$ is isomorphic to $C_{2} \times D_{4}$.

\section{Summary}

In Table 1 is shown a list of the spherical dihedral f-tilings whose prototiles are a spherical kite and an isosceles spherical triangle, $K$ and $T$, of internal angles $\left(\alpha_{1}, \alpha_{2}, \alpha_{1}, \alpha_{3}\right)$, and $(\beta, \gamma, \gamma)$, respectively, in cases of adjacency $I V, V$ and $V I$. Our notation is as follows:

- $\gamma_{1}$ is the solution of equation (2.1), with $\alpha_{2}=\frac{\pi}{2}, \alpha_{1}=\pi-\gamma_{1}$ and $\alpha_{3}=\beta=\frac{\pi}{3} ; \beta_{1}$ is the solution of equation (2.1), with $\alpha_{2}=\frac{\pi}{2}, \alpha_{1}=\pi-\gamma$ and $\alpha_{3}=\pi-2 \beta_{1}$; $\beta_{2}$ is the solution of equation (2.1), with $\alpha_{2}=\frac{\pi}{2}, \alpha_{1}=\pi-\beta_{2}$ and $\alpha_{3}=\gamma=\frac{\pi}{3} ; \gamma_{2}$ is the solution of equation (4.1), with $\alpha_{2}=\frac{\pi}{2}, \beta=\frac{\pi}{3}, \alpha_{1}=\pi-\gamma_{2}$ and $\alpha_{3}=\pi-2 \gamma_{2}$; $\gamma_{3}$ is the solution of equation (4.1), with $\alpha_{2}=\beta=\frac{\pi}{2}, \alpha_{1}=\pi-\gamma_{3}$ and $\alpha_{3}=\pi-2 \gamma_{3}$.

- $|V|$ is the number of distinct classes of congruent vertices;

- $N_{1}$ is the number of triangles congruent $T$ and $N_{2}$ is the number of kites congruent to $K$ (used in the dihedral f-tilings);

- $G(\tau)$ is the symmetry group of each tiling $\tau \in \Omega(K, T)$.

\begin{tabular}{|c|c|c|c|c|c|c|c|c|c|}
\hline f-tiling & $\boldsymbol{\alpha}_{\mathbf{1}}$ & $\boldsymbol{\alpha}_{\mathbf{2}}$ & $\boldsymbol{\alpha}_{\mathbf{3}}$ & $\boldsymbol{\beta}$ & $\boldsymbol{\gamma}$ & $|V|$ & $N_{1}$ & $N_{2}$ & $G(\tau)$ \\
\hline \hline $\mathcal{L}$ & $\pi-\gamma_{1}$ & $\frac{\pi}{2}$ & $\frac{\pi}{3}$ & $\frac{\pi}{2}$ & $\gamma_{1}$ & 3 & 24 & 24 & $C_{2} \times S_{4}$ \\
\hline $\mathcal{J}$ & $\frac{2 \pi}{3}$ & $\frac{\pi}{2}$ & $\pi-2 \beta$ & $\beta_{1}$ & $\frac{\pi}{3}$ & 4 & 48 & 24 & $C_{2} \times S_{4}$ \\
\hline $\mathcal{M}$ & $\pi-\beta_{2}$ & $\frac{\pi}{2}$ & $\frac{\pi}{3}$ & $\beta_{2}$ & $\frac{\pi}{3}$ & 4 & 48 & 24 & $C_{2} \times S_{4}$ \\
\hline $\mathcal{N}$ & $\pi-\gamma_{2}$ & $\frac{\pi}{2}$ & $\pi-2 \gamma_{2}$ & $\frac{\pi}{3}$ & $\gamma_{2}$ & 3 & 48 & 24 & $C_{2} \times S_{4}$ \\
\hline $\mathcal{P}$ & $\pi-\gamma_{3}$ & $\frac{\pi}{2}$ & $\pi-2 \gamma_{3}$ & $\frac{\pi}{2}$ & $\gamma_{3}$ & 3 & 16 & 8 & $C_{2} \times D_{4}$ \\
\hline
\end{tabular}

Table 1: Combinatorial structure of dihedral $\mathrm{f}$-tilings of $S^{2}$ by spherical kites and isosceles triangles in cases of adjacency $I V, V$ and $V I$

\section{References}

[1] C. Avelino and A. Santos, Spherical and planar folding tessellations by kites and equilateral triangles, Australasian Journal of Combinatorics, 53 (2012), 109-125. 
[2] C. Avelino and A. Santos, Spherical Folding Tessellations by Kites and Isosceles Triangles: a case of adjacency, Mathematical Communications, 19 (2014), 1-28.

[3] C. Avelino and A. Santos, Spherical Folding Tessellations by Kites and Isosceles Triangles II, International Journal of Pure and Applied Mathematics, 85 (2013), 45-67.

[4] C. Avelino and A. Santos, Spherical Folding Tessellations by Kites and Isosceles Triangles III, submitted.

[5] A. Breda, A class of tilings of $S^{2}$, Geometriae Dedicata, 44 (1992), 241-253.

[6] A. Breda and P. Ribeiro, Spherical f-tilings by two non congruent classes of isosceles trianglesI, Mathematical Communications, 17 (2012), 127-149.

[7] A. Breda and A. Santos, Dihedral f-tilings of the sphere by spherical triangles and equiangular well centered quadrangles, Beiträge zur Algebra und Geometrie, 45 (2004), 447-461.

[8] A. Breda and A. Santos, Dihedral f-tilings of the sphere by rhombi and triangles, Discrete Mathematics and Theoretical Computer Science, 7 (2005), 123-140.

[9] A. Breda, P. Ribeiro and A. Santos, A Class of Spherical Dihedral F-Tilings, European Journal of Combinatorics, 30 (2009), 119-132.

[10] R. Dawson, Tilings of the sphere with isosceles triangles, Discrete and Computational Geometry, 30 (2003), 467-487.

[11] R. Dawson and B. Doyle, Tilings of the sphere with right triangles I: the asymptotically right families, Electronic Journal of Combinatorics, 13 (2006), \#R48.

[12] R. Dawson and B. Doyle, Tilings of the sphere with right triangles II: the $(1,3,2),(0,2, n)$ family, Electronic Journal of Combinatorics, 13 (2006), \#R49.

[13] S. Robertson, Isometric folding of riemannian manifolds, Proc. Royal Soc. Edinb. Sect. A, 79 (1977), 275-284.

[14] Y. Ueno and Y. Agaoka, Classification of tilings of the 2-dimensional sphere by congruent triangles, Hiroshima Mathematical Journal, 32 (2002), 463-540. 